\title{
Biotechnological applications of mammalian odorant-binding proteins
}

\author{
Filipa Gonçalves (D), Artur Ribeiro (D), Carla Silva (D) and Artur Cavaco-Paulo (D) \\ Centre of Biological Engineering, University of Minho - Campus de Gualtar, Braga, Portugal
}

\begin{abstract}
The olfactory system of mammals allows the detection and discrimination of thousands of odors from the environment. In mammals, odorant-binding proteins (OBPs) are considered responsible to carry odorant molecules across the aqueous nasal mucus to the olfactory receptors (ORs). The three-dimensional structure of these proteins presents eight antiparallel $\beta$-sheets and a short $\alpha$-helical segment close to the $C$ terminus, typical of the lipocalins family. The great ability of OBPs to bind differentiated ligand molecules has driven the research to understand the mechanisms underlying the OBP function in nature and the development of advanced biotechnological applications. This review describes the role of mammalian OBPs in the olfactory perception, highlighting the influence of several key parameters (amino acids, temperature, ionic strength, and $\mathrm{pH}$ ) in the formation of the OBP/ligand complex. The information from the literature regarding OBP structure, affinity, the strength of binding, and stability inspiring the development of several applications herein detailed.
\end{abstract}

\section{ARTICLE HISTORY}

Received 8 September 2020

Revised 28 October 2020

Accepted 15 November 2020

\section{KEYWORDS}

Lipocalin; odorant-binding protein (OBP); binding; ligand; fragrance; odorant molecules; protein; structure; liposomes

\section{Introduction}

The olfactory system of mammals belongs to the chemical senses (smell, taste) playing a crucial role in the detection and presentation of different odorant molecules from the environment, translating them into varied perceptions and behaviors. This system includes odorant-binding proteins (OBPs), for the binding and presentation of odors, and olfactory receptors (ORs) located in olfactory sensory neurons, associated with the olfactory bulb, for the detection and discrimination of the odors $[1,2]$.

\section{Mammalian olfactory transduction system}

The olfactory system of mammals is of vital importance in the identification of odorants; in prey/predators' relations; for reproduction purposes and the identification of toxic foods [3-5]. Olfactory perception is initiated when an odorant, existent in the air, is presented and interacts with the olfactory receptors (ORs) of the olfactory sensory neurons, through the nasal mucus of vertebrates $[2,6,7]$. This smelling process relies in a synergistic mechanism between the odorant, the odorant-binding proteins (OBPs), and the olfactory receptors (ORs) $[3,7]$. The odorants are usually low molecular weight, hydrophobic, and volatile molecules that cross the hydrophilic nasal mucus to reach the ORs in a process mediated by the OBPs $[4,7]$. The OBP/odorant complex is recognized by the OR a protein belonging to the subfamily class $A$ of a $G$ protein-coupled receptor (GPCR), existent in the olfactory sensory neurons [8]. The trigger of OR leads to an intracellular signaling cascade resulting in the production of guanosine diphosphate (GDP) on the $\alpha$ subunit of the G-protein. The activated $\alpha$ subunit interacts with the adenylyl cyclase (AC) that converts adenine triphosphate (ATP) into cyclic adenine monophosphate (CAMP). This signaling cascade induces the inflow of $\mathrm{Ca}^{2+}$ and $\mathrm{Na}^{+}$ions by activating the membrane channels. The consequent increase in the internal concentrations of $\mathrm{Ca}^{2+}$ causes the opening of $\mathrm{Ca}^{2+}$-activated $\mathrm{Cl}^{-}$channels that produce an efflux of $\mathrm{Cl}^{-}$from the neuronal cilia, contributing to olfactory neuron membrane potential depolarization. The depolarization leads to action potentials that are transmitted along the axon of the olfactory sensory neuron until it reaches the olfactory bulb followed by interpretation of olfactory signal in different areas of the brain (piriform cortex, olfactory tubercle, anterior olfactory nucleus, and specific parts of the amygdala and entorhinal cortex) [1,2,9-11] (Figure 1). 


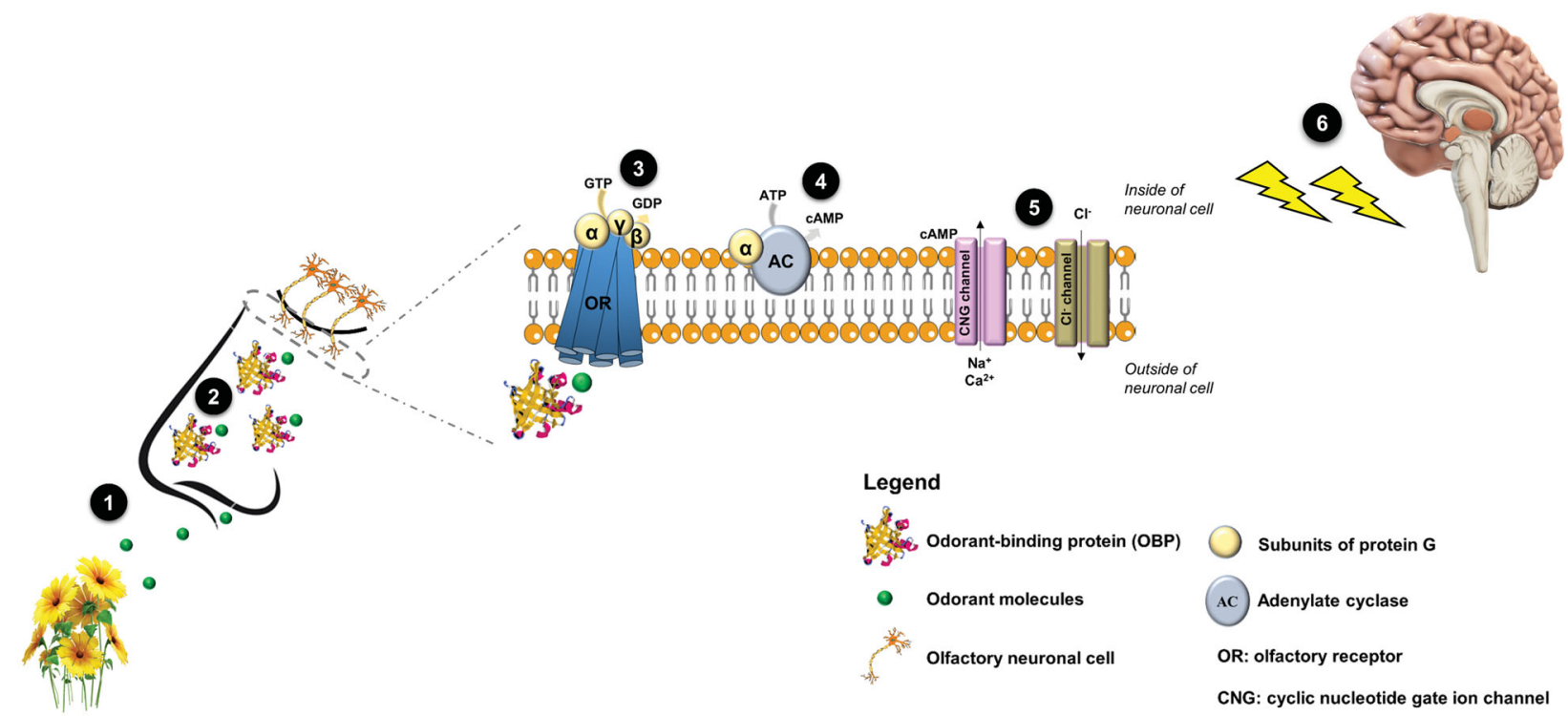

Figure 1. Mechanisms of mammalian olfactory systems. In the presence of an odorant molecule (1), the odorant-binding proteins (OBPs) present in the nasal mucus carry it to the olfactory receptors (ORs) present in olfactory neuronal cells (2). Consequently, an intracellular signaling cascade is trigged via G-protein (3), resulting in the production of cyclic nucleotide (cAMP) by the activation of adenylyl cyclase (AC) (4). cAMP induces the inflow of $\mathrm{Ca}^{2+}$ and $\mathrm{Na}^{+}$ions which increases inside of neuronal cell causes an efflux of $\mathrm{Cl}^{-}(5)$, contributing to the olfactory neuron depolarization. The depolarization of the olfactory neuron membrane leads to modification in action potential that is conducted along the axon until achieve the olfactory bulb and the olfactory signal is interpreted by the brain (6). Figure was created based on Sankaran et al. [12].

\section{The role of mammalian odorant- binding proteins}

The odorant-binding proteins (OBPs) are small ( $\sim 15$ to $20 \mathrm{kDa}$ ), extracellular, water-soluble proteins and members of the lipocalins superfamily [13-16]. OBPs are expressed in high concentrations in the glands of the nasal mucosa and in the vomeronasal organ being released into the nasal mucus of vertebrates $[12,15,17]$. Their expression occurs momentarily after birth for the vertebrates, reaching their maximum levels within 2-3 d [18]. During their lifetime, the OBPs are not produced at a constant rate, they are only expressed in certain periods and under a variety of physiological conditions [17].

The first vertebrate OBP identified was isolated in 1985, from the nasal mucosa of bovines [19]. In the last three decades, OBPs from other species, including pigs [20], rabbits [21], elephants [22], mice [23], rats [24], insects [25], frogs [26], and humans [27], have been identified.

Although the exact function of OBPs remains unclear, studies have been postulated that these proteins are involved in several processes related to the binding and presentation of odors. Once OBPs are secreted into the nasal mucous and present capacity to bind different odors, it is believed that they are responsible to transport hydrophobic odorant molecules, in their calyx-shaped cavity, across the aqueous mucus barrier toward the olfactory receptors [1,5,16,28-30]. They also might be involved in the termination of the olfactory signal by removing odorants from the olfactory receptors after stimulation [5,31,32]. Furthermore and according to Ikematsu et al. [33], OBPs can be also involved in general defense mechanisms in mammals, especially in the removal of harmful substances present in breathed air, thus maintaining the receptor binding sites in a state of readiness [34]. Grolli et al. [33] investigated the binding properties of OBPs to the 4-hydroxy2-nonenal (HNE), a reactive lipid peroxidation end-product. With this study, they intended to establish a functional relation between the OBPs and the molecular mechanisms involved in the combat to free radical cellular damage. HNE is produced through the peroxidation of unsaturated fatty acids by cells as a consequence of the exposure to the oxygen present in the inhaled air. The binding data revealed a dissociation constant (Kd) of 4.9 and $9.0 \mu \mathrm{M}$ for porcine and bovine OBP, respectively [33]. This constant evaluates the strength of the interactions between the ligands and the proteins. Low Kd values are observed by steeper slopes (fluorescence vs ligand concentration) and correspond to the greater binding affinity of the ligand to OBP; high Kd values mean weak ligand affinity. These preliminary results suggest that OBP can also be associated with the reduction of HNE toxicity in the nasal mucosa [33]. This discovery helps to understand the 

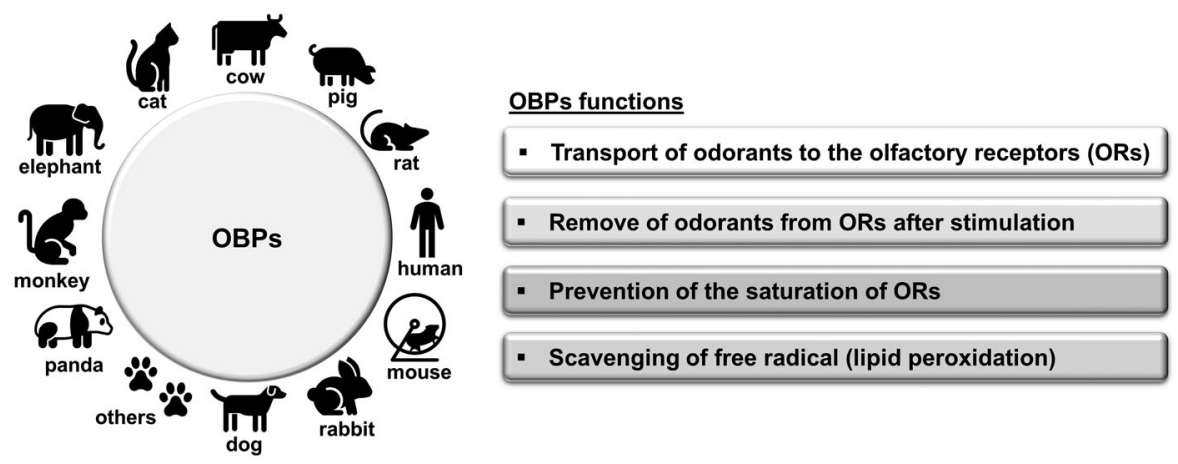

Figure 2. Mammalian OBPs origin (left) and their function in the olfactory system (right). Figure was created based on GomezVelasco et al. [17], Grolli et al. [33], Ikematsu et al. [34], Muthukumar et al. [5], and Pelosi et al. [29].

function of the OBP in the protection of the nasal mucosa, exposed to airflow and associated oxidative stress. Some authors refer that ORs have the ability to be stimulated by odors, even in the absence of OBPs $[35,36]$. However, the results did not explain why the presence of OBP improved the affinity of the OBP/odor complex by the receptor. Vidic et al. [37] demonstrated that high concentrations of helional odorant, an aldehyde used as a perfume in soap and laundry detergents when in the absence of the native rat OBP-1 resulted in a significant diminution of the response of OR17-40 to helional [37]. Furthermore, when the ligand concentration is high, OBPs are crucial to prevent the saturation of ORs binding sites [29,37-39]. Figure 2 resumes the main functions of OBPs and some of the organisms from where they were identified.

Some OBP isoforms have been identified within the same species. These isoforms diverge in their amino acid sequences and in their specificity to different types of ligands [40]. For example, there are several OBP isoforms in rats that differ in their binding preference [41]. Rat OBP-1 preferentially binds to heterocyclic compounds, such as pyrazine derivatives, whereas OBP-2 presents more specificity for carboxylic acids and longchain aliphatic aldehydes. On the other hand, rat OBP-3 appears to have a strong interaction with odorants composed of saturated or unsaturated ring structures [40,41].

The OBPs from different species, share low sequence similarity. For example, pig OBP-I (pOBP) and human OBP ( $\left(\mathrm{hOBP}_{11 \mathrm{a}}\right.$ ) share only $13.9 \%$ of sequence similarity, while $\mathrm{pOBP}$ and bovine OBP (bOBP) share $42.7 \%$ [28,42]. Intra OBP species analysis, for example, testing two human OBPs sequences ( $\mathrm{hOBP}_{\| \mathrm{la}}$ and $\mathrm{hOBP}_{\mathrm{Ilb}}$ ) displayed $95 \%$ of identity [43], despite that hOBP expressed in the nasal mucus, saliva, and lachrymal glands; while hOBP ${ }_{\| \mathrm{bb}}$ is expressed in the genital organs. A low number of $3 \mathrm{D}$ structures of OBP proteins are available, as we confirmed in Protein Data Bank (PDB).
Regarding the protein sequences predicted as OBPs, through blast analysis, we retrieved several sequences from PDB, UniProt (Universal Protein), and Ensembl genome browser [44]. In order to better understand the similarities between OBPs from different species, a cladogram was created (Figure S1). Since this review is focused on mammalian OBPs and due to the high number of sequences observed (less sensitivity and low similarity), the sequences of birds, reptiles, and bony fishes were rejected. All different sequences, even from the same species, were considered to construct the cladogram. The results show that all mammalian species are well distributed in different orders.

\section{Physicochemical and structural properties of OBPs}

In the last two decades, several structures of OBPs have been solved by $\mathrm{X}$-ray diffraction and deposited in Protein Data Bank (PDB). However, a low number of 3D structures are available. In 1996, a OBP structure isolated from bovine (Bos taurus) nasal mucosa at $2.0 \AA$ of resolution (PDB ID: 1OBP) was reported $[45,46]$. Spinelli et al. [47] obtained the crystallographic structure of pig (Sus scrofa) OBP with $2.25 \AA$ of resolution (PDB ID: 1A3Y) [47]. Actually, there is a more accurate structure of pOBP with $1.48 \AA$ of resolution (PDB ID: 1DZK) [48]. Also, White et al. [39] reported the crystal structure of rat OBP-1 at $1.6 \AA$ resolution (PDB ID: $3 \mathrm{FIQ}$ ) and the monomeric state of this protein, either in the crystalline form or in solution, when under native conditions [39]. Crystal structure of the human OBP, designated as OBP Ila, was also obtained (PDB ID: 4RUN) [49]. The protein sequences of these OBPs are very different, as we can observe in the alignment of the sequences in Figure S2A. The structures of the OBPs available in PDB are presented in Figure S2B. Despite many other OBP sequences are known (total or partially) their structure is not yet resolved. 


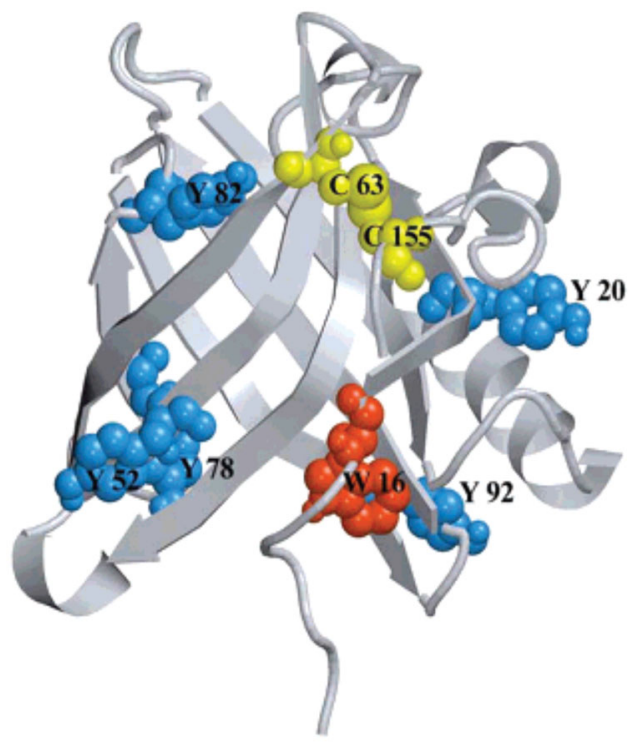

Figure 3. Cartoon diagram of porcine OBP protein (pOBP). The figure indicates the localization of Trp16 residue (W16), five Tyr residues (Y20, Y52, Y78, Y82, and Y92), and the disulfide bridge between Cys63 and Cys155. The figure was retrieved from Staiano et al. [53], constructed on the basis of pOBP structure given in file 1A3Y.pdb.

Despite the genetic variability between OBPs from different mammalian species, lipocalin members present some characteristics that allow their identification. For example, their tertiary structure is well conserved, with the $\beta$-barrel structure composed of eight $\beta$-strands (designed by $A$ to $H$ ) linked by seven loops (L1-L7) and connected to a short $\alpha$-helix close to the $\mathrm{C}$-terminus and a ninth $\beta$-strand followed by the disordered C-terminal tail $[47,50,51]$. Fourier-transform infrared spectroscopy (FT-IR) spectrum of pig OBP suggested the existence of $51 \% \beta$-sheets and $8 \% \alpha$-helix corroborating the values observed by X-ray diffraction $(43 \%$ and $10 \%$, respectively) [52]. Vertebrate OBPs have other conserved characteristics that allow their identification: a GxW motif (G: glycine; $x$ can be any residue; W: tryptophan) on the N-terminus (residues 14-16, in pOBP); a glycine residue at the C-terminus (Gly119, in pOBP); and two cysteines in the middle and at the C-terminal end of the sequence [42]. In porcine, the conserved Cys63, and Cys155, forms a disulfide bridge tightening the flexible $C$-terminal $\alpha$-helix domain and the $\beta$-barrel (Figure 3) [39]. In addition, an $Y x x x Y x G$ motif can also be found (residues 78-84, in pOBP). Negatively charged residues (Glu and Asp) are also systematically present at positions 46, 130, 143, and 153 in pOBP. In OBPs, except for Asn145, there are some aromatic and aliphatic residues or glycine completely conserved (Phe55, Phe88, Phe132, Ile104, Ile141, Ile146, Gly109, Gly119, and Gly140 in pOBP) [28].
Bovine OBP (bOBP) differs from the other lipocalins by lacking the conserved disulfide bond and by their ability to form domain-swapped dimers [45,54]. bOBP was the first mammalian OBP with a crystal structure solved and the analysis of this structure revealed a domain-swapped dimer, in which the helix near the Cterminal region of each monomer is packed against the $\beta$-barrel of the other $[45,46]$. The domain swapping mechanism of this OBP was explained by the absence of a glycine residue at the hinge region linking the $\beta$-barrel to the $\alpha$-helix and also by the lack of the disulfide bridge [55]. A bovine mutant OBP obtained by the insertion of a glycine residue in the position 121 of bOBP, and by the replacement of W64C and H155C (GCC-bOBP), resulted in a monomeric protein without any structure perturbation revealing the importance of the absence of the Gly121 and cysteine residues during the formation of the dimeric structure [54]. Experimental and computational data also revealed that bOBP is a dimer at neutral $\mathrm{pH}$, contradicting the monomeric structure at $\mathrm{pH} 2.5$. Acid $\mathrm{pH}$ results in the loss of the swapped dimeric conformation, without alteration of the tertiary and secondary structures [56].

The structure of mammalian OBPs proved to be highly stable and resistant to degradation by temperature, organic solvents, $\mathrm{pH}$ variation, or proteolytic digestion $[29,30]$. Vertebrate OBPs can resist at high temperatures before undergoing denaturation, and, if unfolding occurs, this phenomenon can be reversed after restoring the initial conditions [52]. The FT-IR spectra for $\mathrm{POBP}$ revealed a structure exceptionally stable to thermal denaturation (up to $80^{\circ} \mathrm{C}$ ), particularly in the presence of the 2-isobuthyl-3-methoxypyrazine and 3,7dimethyl-1-octanol ligands [52]. The FT-IR data also showed two transition phases occurring at $65-70^{\circ} \mathrm{C}$ and $80-85^{\circ} \mathrm{C}$, related to molten globule states of the $\beta$-barrel, maintaining however the structural integrity at such temperatures [52]. Besides, circular dichroism spectroscopy measurements proved that the pOBP preserves a structural stability up to $65^{\circ} \mathrm{C}$ [57]. Exceptionally, bOBP maintains the dimer form even in the presence of $1.5 \mathrm{M}$ of guanidinium chloride $(\mathrm{GdnHCl})$ [58]. Also pOBP is only completely unfolded when dissolved in solutions containing $3.5 \mathrm{M}$ of $\mathrm{GdnHCl}$ [53] or when it is suspended in $8 \mathrm{M}$ of urea, as verified by circular dichroism spectroscopy [59].

\section{Binding affinity and selectivity of mammalian OBPs}

The first mammalian OBP was discovered in the nasal mucosa of bovines, by ligand-binding experiments 
using a radioactive pyrazine ligand $\left({ }^{3} \mathrm{H}\right]-2$-isobutyl-3methoxypyrazine) $[19,60]$. The first OBPs binding assays were based on methods involving radioactive-labeled ligands and included the separation of bounds and free ligands by electrophoresis, gel filtrations, or other techniques $[29,60,61]$. These ligand-binding experiments were used to measure the affinity of odorants toward OBPs and were improved over time, replacing the radioactive probes with fluorescent ligands [61]. Three approaches can be used to measure the binding of a ligand to OBP. The first is based on centrifugation steps where the free ligand is separated and quantified. The second uses a dialysis membrane to separate the OBP/ ligand complex from the free ligand. In these two first approaches, the binding corresponds to the difference between the initial amount of ligand added to the binding reaction and the free ligand measured after separation. The third approach directly determines the formation of the OBP/ligand complex using a fluorescent ligand, without any separation step. This method, is simple, fast, and only requires a small amount of protein and no separation step is needed, avoiding though the error measurements related to this last step [61]. Several fluorescent ligands are actually used to measure the OBP-mediated binding: 6-ptoluidinylnaphthalene-2sulfonate (TNS), 2-isobutyl-3-methoxypyrazine (IBMP), $\mathrm{N}$-phenyl-1-naphthylamine (1-NPN), fluorescein and 1-aminoanthracene (1-AMA) [29,62]. The 1-AMA was used for the first time to measure the activity of pig OBP (OBP-I) [52] being actually widely applied to study the interactions of the lipocalin superfamily proteins (Table S1). The free and the 1-AMA bound to pig OBP-I (OBP/AMA complex) can be quantified by measuring the fluorescence with excitation wavelength at $295 \mathrm{~nm}$. The maximum wavelength of 1-AMA is shifted from 537 to $481 \mathrm{~nm}$ when 1-AMA is bound to pig OBP $[52,63,64]$.

The affinity of non-fluorescent ligands to OBPs can be accessed by their ability to compete with the fluorescent ligand. In the competitive binding experiments, the protein is incubated with the fluorescent ligand at a fixed concentration, and then increasing amounts of a non-fluorescent ligand are added $[61,63]$. The strength of the non-fluorescent ligand to bind to the OBP and replace the fluorescence probe is directly related to the affinity of this compound to the protein. Competitive assays have revealed that pig OBP has a capacity to bind a variety of different structural ligands including terpenoids (3,7-dimethyloctanol, carvone, $\beta$-citronellol), linear aldehydes (decanal, vanillin), and aromatic compounds (benzyl benzoate, coumarin) $[29,48,65,66]$. The authors in Tegoni et al. [28] refers that the best ligands present dissociation constants in the interval of
$0.1-1.0 \mu \mathrm{M}[17,28]$. However, the range of values that are considered low or high dissociation constants are not clearly defined by the scientific community. Furthermore, the binding affinity can be reported by an association constant $(\mathrm{Ka})$ that represents the inverse of the $K d$ value $(K a=1 / K d)$ [67]. A list with the distinct dissociation constants, that is, the binding capacity of the mammalian wild-type and mutated OBPs is indicated in Table S1. More recently, derived fluorescent compounds (phenylnaphthalen-1-amine (1-NNN) and 2naphthylamine (2-NNN)), were synthesized and used to measure the binding property of OBPs [68]. These new probes present an excitation and emission wavelength in the visible region of the spectrum (Table S1, mutant pOBP-F88W). Besides, these probes are useful to create a simple, accurate, and sensitive optical sensor for odorants [68], only a few studies have reported their use to measure the binding of ligands to OBPs.

\section{Mechanistic insights about the OBP mode of action}

The formation of the OBP/ligand complex is dependent on the type of ligand, the ligand's proximity to the OBP protein, the OBP structure, and the size of the OBP cavity [68-70]. During complex formation, different amino acidic residues of OBP may be involved in the binding process. Site-directed mutagenesis, recombinant technologies, advances in protein sequencing, and the use of different bioinformatic tools, allowed the identification of the amino acids and the interactions involved in the binding process. Molecular dynamic (MD) simulations and docking analysis allow one to calculate the coordinates of $\mathrm{C} \alpha$ atoms of protein variations; the thermodynamics and energies involved in binding; and to predict the structure of OBP with and without ligand. Furthermore, using MD simulations it is possible to infer and quantify the percentages of secondary structure elements of OBPs; evaluate the solvent accessibility of amino acid residues, and identify the physicochemical interactions involved in the binding process. Together with site-directed mutagenesis, this tool helps the identification of the residues involved in the OBP/ligand interaction $[64,69,71,72]$.

\section{The nature of the ligand influences the binding site at the OBP surface}

OBPs are known for their capacity to bind ligands with different sizes and functional groups. Several studies have been conducted to understand the binding process and to identify which residues on the OBP's 
binding site interact with the ligands during the formation of the OBP/ligand complex. While some ligands seem to interact with "universal" residues of the OBP binding site, others bind in a more specific manner. The nature of the ligand seems to influence the binding process and to determine the amino acids of OBP involved in the binding. In pig OBP, the highly conserved Tyr82 residue has been identified as being involved in the binding process of several ligands. The replacement of this amino acid present in the protein binding pocket, by another residue, with the same physicochemical properties, like phenylalanine, resulted in any alteration on the cavity structure, which is consistent with the assigned function [73]. Notwithstanding, the substitution of the same residue by an alanine (small hydrophobic amino acid), resulted in a structural alteration of the binding pocket, supporting the involvement of Tyr82 in the binding process [73]. Meillour et al. [74] demonstrated experimentally that Phe35 and Tyr82, both located in the pOBP pocket, are involved in the binding and release of 1-AMA (aromatic) and of undecanal (UND, aldehyde) ligands [74]. The authors observed that the fluorescence spectra for the pOBP-Phe35Ala and pOBP-Tyr82Ala mutants with increased concentrations of 1-AMA presented a very similar fluorescent value, and are different from wildtype pOBP. This result indicates that both aromatic residues, Phe35 and Tyr82, are necessary to retain the ligand in the binding pocket of pOBP. In the double mutant pOBP-Phe35Ala/Tyr82Ala, the maximum emission fluorescence did not shift upon 1-AMA addition, indicating the absence of 1-AMA binding toward the OBP mutant [74]. Molecular dynamic (MD) simulations supported the reported experimental data. The binding process is initiated by the opening of the OBP pocket site through the shift of tyrosine and phenylalanine residues [42]. MD simulations report that the interaction between pig OBP and the ligand occurs through the shift of the residues mainly located at the junction between the $\beta$-strands $D$ and $E$ and the L1 and L5 loop (Figure S3) [75].

The number of interactions and the residues involved between OBP and the different odorants has been found to vary between two and ten residues. For example, in pOBP, the lle21, Met39, Val80, Tyr82, Phe88, Ile100, Asn102, Met114, Gly116, and Leu118 residues are involved in the interaction with benzophenone (BZP, aromatic ketone) [48]. For benzyl benzoate (BZB) the residues involved in the binding are described to be Asn86, Phe88, Asn102, Met114, and Thr115 [48,76]. The 2,6-dimethyl-7-octen-2-ol (DHM), the undecanal (UND, aldehyde), and the 2-isobutyl-3-metoxypyrazine
(IBMP) are oriented differently in the cavity of the protein and interact with different amino acid residues (Asn86, Asn102, Try82, Phe35, Phe55, Lys28, and Asp110) $[48,74,75]$. While for some ligands, several amino acid residues are involved in the ligand interaction, for others, few residues participate on the process. For example, the binding of thymol to pOBP seems to be mediated by only two residues, the nonpolar Ile21 and Phe88 [48]. Table S2.1 presents the residues identified as being involved in the binding of wild-type pig OBP to the specific ligand.

A recent study, performed by our group, revealed that the binding conditions, like temperature, could also determine the residues involved in the binding process. In this study, we designed two mutant proteins. A truncated pig OBP (tOBP), resulted from the replacement of two phenylalanine residues at the binding pocket of OBP-I (F44A and F66A) and from the deletion of the first 16 residues of the $\mathrm{N}$-terminal. Also, the $\mathrm{OBP}:: \mathrm{GQ}_{20}:: \mathrm{SP}-\mathrm{DS} 3$, resulted of the fusion of pig OBP-I with the anchor peptide SP-DS3 (DRDDQAAWFSQY) and a spacer with 20 repetitions of glycine-glutamine residues $\left(\mathrm{GQ}_{20}\right)$ [69]. Using the AutoDock Vina we predicted the docking positions of 1-AMA in the new OBPs at different binding temperatures $\left(25\right.$ and $37^{\circ} \mathrm{C}$ ). In this study, we have identified some residues previously reported (Phe35, Phe55, Tyr82, Asn86, Asn102, and Met114) for the wild-type pig OBP while others were identified and reported for the first time. For the tOBP protein, the Ala83 and Asn104 amino acids were identified for the first time as being involved in the binding at $25^{\circ} \mathrm{C}$, while Val147 and lle149 were identified, here in this study, as being involved in the 1-AMA binding, at $25^{\circ} \mathrm{C}$. For $\mathrm{OBP}:: \mathrm{GQ}_{20}:: \mathrm{SP}-\mathrm{DS} 3$, the amino acids Asn32, Pro34, Lys58, and Arg152 at $25^{\circ} \mathrm{C}$ and the Asn104, Val105 and Asp106 were also reported, for the first time, as being involved in the 1-AMA binding at $37^{\circ} \mathrm{C}[69]$.

Docking simulations of benzene to porcine OBP (PDB ID: 1DZK) identified some residues involved in the binding process: lle21, Phe35, Asn102, Met114, Thr115 and Gly116, polar and non-polar residues [57], which were also identified by us [69]. The same authors identified the residues involved in the binding of benzene to bovine OBP: Phe89, Ala101, Asn103, Leu115, Thr116, and Phe119, polar and non-polar residues [57].

Bianchet et al. showed that in bovine OBP (bOBP), the access of a ligand to the binding cavity of the protein is controlled by the hydrophobic aromatic Phe89, through rotation around a carbon-carbon single bond [46]. Phe89, but also Phe54, seems to control access to the bOBP pocket [28]. Hajjar et al. [77] showed, using 
MD simulation, that the hydrogen bonding between the aromatic polar Tyr20 and Tyr78 residues increases in the presence of the thymol ligand. Tyr83 (conserved tyr82 in pOBP) was also referred to as being situated near the entrance of the binding cavity as demonstrated by MD simulations. This amino acid constitutes and regulates the entrance to the bOBP cavity $[75,77]$. Moreover, Tyr83 appears to be especially involved in the unbinding process and can be considered as the gate of OBPs' binding pocket. This amino acid is highly conserved among the members of the lipocalin superfamily, indicating its high relevance to the binding process [75]. Other interaction studies, revealed that Phe36 and Tyr83 residues are responsible to regulate access to the binding site in bOBP, by the rotation of residues side chains, opening the barrel entrance, and increasing the binding cavity volume [56]. Table S2.2 presents the residues identified as being involved in the binding of some ligands to the bovine OBP.

Studies with OBPs from other mammals have also been performed, in order to understand which amino acids are involved in the binding process with different ligands. Human variant $\mathrm{hOBP}_{\text {Ila }}$ presents an affinity to numerous odorants, including aldehydes and fatty acids. The binding pocket of this OBP presents three lysine residues (Lys62, Lys82, and Lys112). Using sitedirected mutagenesis and fluorescent probes, Lys 112 showed to be essential for the specificity of hOBP ${ }_{\text {Ila }}$ for aldehydes and small carboxylic acids, like undecanal (UND), N-phenyl-1-naphthylamine (1-NPN), and 11-(5(dimethylaminonaphthalenyl-1-sulfonyl)-amino) undecanoic acid (DAUDA) [78] (Table S2.3). In the giant panda, the pocket cavity of AimelOBP3, is shielded from solvents by three residues: Asp87, Asn90, and Met39. The authors made site-directed mutagenesis and docking simulations of Asn90Leu (mutation of a polar residue by non-polar residue), and verified a reduction in the affinity to linear aldehydes, while affinity to terpenoids was maintained [79]. Other mutations, like Glu120Ala, drastically reduced the protein affinity, while Ser122Ala (substitution of a polar residue by non-polar residue) did not reveal any effect on the affinity properties of the protein. Furthermore, docking simulations confirmed that Asn90 and Glu120 are important residues in the binding ability of this protein [79] (Table S2.3).

The buffalo nasal OBP model created by Muthukumar et al. [5] showed 30 possible binding sites, capable to accommodate different odorant molecules [5]. Table S2.4 presents the residues identified as being involved in the binding of different ligands toward the buffalo OBP.

\section{Understanding the mammalian OBP-odorant interactions}

Binding affinity is influenced by non-covalent intermolecular interactions such as: hydrogen bonding, electrostatic interactions, hydrophobic and van der Waals interactions, between the ligand and the OBP [67]. Docking experiments using pig and bovine OBPs, studied the interactions involved in the binding of benzene to the proteins. In this study, the authors identified hydrophobically and van der Walls interactions as the predominant interactions involved in the binding process [57]. The binding process between OBP and the ligands involves other physicochemical interactions, such as hydrogen and ionic bonds, as well as van der Waals interactions $[28,80]$.

Molecular dynamic (MD) simulations revealed significant flexibility of the OBP in the presence of different ligands [77]. Using MD simulation, it was shown that the interaction between 2,6-dimethyl-7-octen-2-ol (DHM) ligand and pOBP was driven by van der Waals interactions with binding energy around $32 \mathrm{kcal} / \mathrm{mol}$, for the first $1700 \mathrm{ps}$ of simulation. However, for later simulation times, there was an increase in the total interaction energy to $42 \mathrm{kcal} / \mathrm{mol}$. This increase is justified by the formation of hydrogen bonds between the ligand and the Asn86 and Asn 102 residues of pOBP $[75,81]$. MD simulations have shown that hydrophobic interactions are dominant in the depths of the binding pocket, and the hydrogen-bonding is important in the interaction between residues at larger distances. Both interactions are responsible for the high stability of the protein at high temperatures $[69,72,77]$.

\section{Applications of mammalian OBPs}

Inspired by the olfactory sense and on the ability of OBPs to interact with several molecules, the exploitation of OBPs in chemosensory design has been encouraged. OBPs present several interesting features such as low molecular weight, high thermal stability, binding ability at different $\mathrm{pH}$ values, high resistance to several organic solvents, and to proteolytic digestion. These features combined with OBPs' high sensitivity, fast response time, reversibility, and capacity to detect odors at very low concentrations, make these proteins excellent candidates to be used in the development of several biotechnological applications $[29,30]$. OBPs can be used to monitor environmental contamination, detecting dangerous substances, hazardous agents, or in the detection of pathogens, pesticides, and drug residues in food $[29,82]$. Also, OBPs show potential to be 


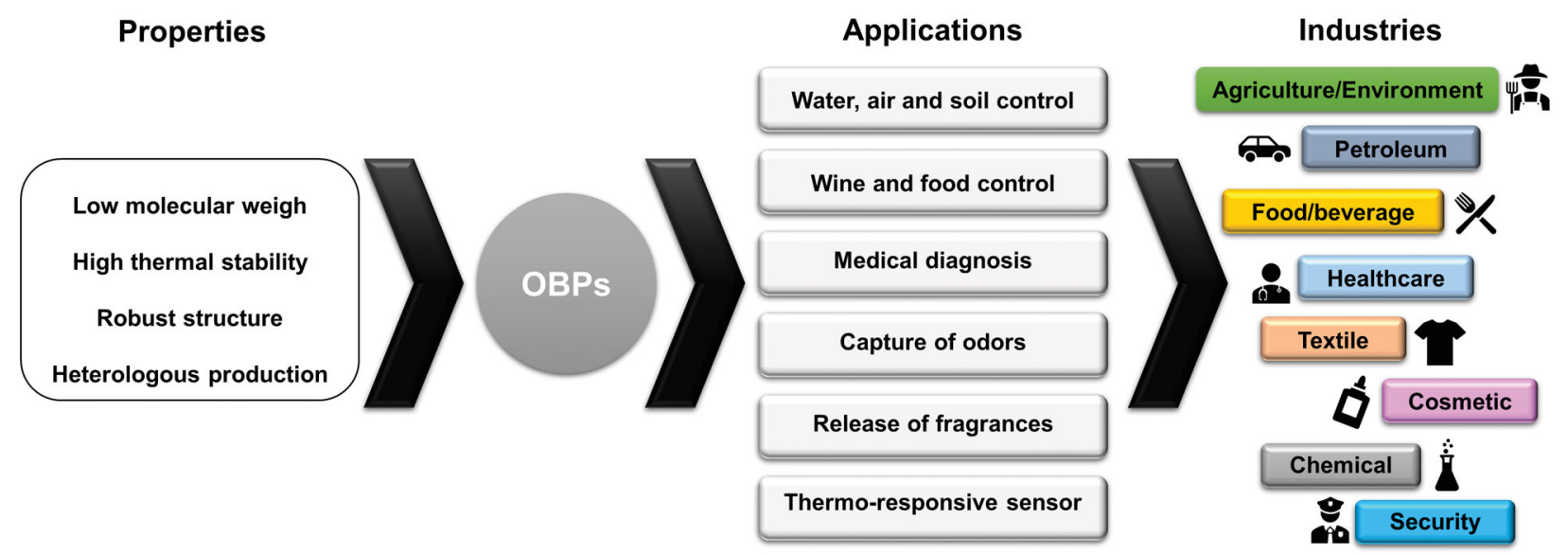

Figure 4. Properties and applications of mammalian OBPs.

used as deodorizers and in medical diagnostics (Figure 4) [12,66].

\section{OBPs as biosensors}

The exceptional stability of OBPs opened the possibility to explore and apply these proteins in several areas. They are considered good candidates for the creation of sensing devices that can provide rapid, sensible, and selective detection of compounds. These new OBPbased sensing devices can be used in agriculture, environmental monitoring, healthcare, military defense, and in the food industry $[29,30,83]$.

Biosensors for control of water, air and soil contamination

Aldehydes are toxic, volatile, polar, and reactive compounds found in contaminated environments resulting from industrial wastes. These compounds represent a threat to public health and thus, their monitoring is of the utmost importance [84]. The traditional detection and quantification of these aldehydes are made by gas chromatography (GC), which implies elevated costs and times of processing and analysis. In a recent study, pig OBP (pOBP) was used for the detection of BTEX pollutants (e.g. benzene, toluene, ethyl-benzene, and xylene isomers) originated by pesticides and petroleum industries [57]. In this study, the authors demonstrated, by molecular docking analysis and experimental data, that benzene has a high affinity $(\mathrm{Kd}=0.30 \pm 0.07 \mu \mathrm{M})$ toward pOBP. Based on this data, pOBP appears to be a good candidate as a biosensor for the detection and removal of benzene from pollutants [57]. Another interesting application of OBPs in the monitoring of VOCs released from plants, which may indicate the plants' health, the level of environmental stresses, and the detection of pesticides [83]. Bianchi et al. [85] created a cartridge-like device, composed of bovine OBP (bOBP) coated on nickel nitrilotriacetic acid (Ni-NTA) agarose resin, taking advantage of the His-tag added to the protein sequence. This cartridge showed a capacity for the removal of triazine herbicides from water [85]. The data obtained for this sensor estimated the capacity to purify $38 \mathrm{~L}$ of water contaminated with $4 \mu \mathrm{g} / \mathrm{L}$ atrazine, using $8 \mathrm{mg}$ of bOBP coupled to $1 \mathrm{ml}$ Ni-NTA agarose [85].

A biosensor proposed by Di Pietrantonio et al. [86], composed of five surface acoustic wave (SAW) resonators coated with wild-type bOBP, double mutant bOBP and wild-type pOBP, was used to detect several chemical molecules. The sensor was able to discriminate the octenol from the R-(-)-carvone vapors, at low detection limits, making it an excellent multisensor for the assessment of food contamination by molds or for the evaluation of indoor air quality. The two bOBPs showed very similar responses to octenol and carvone, while the pOBP gave a much stronger signal for octenol. Furthermore, the sensor presented a robust behavior with good reproducibility and sensitivity in the range of a few ppm [82,86]. Also, Mulla et al. [87] described pOBP-F88W immobilized on SAW resonators coated by a gold surface. The carvone binding was monitored by modification in the electrical property of the protein. The biosensor was capable to discriminate $\mathrm{S}$ $(+)$-carvone and $\mathrm{R}-(-)$-carvone, presenting a high affinity to S-(+)-carvone [87]. In another study, Hou et al. [88], created films with recombinant rat OBP-1F and transferred them to gold electrodes. Using non-faradaic electrochemical spectroscopy, untreated films revealed less electric resistance $(1.18 \mathrm{M} \Omega$ ) than the films exposed to vapors of isoamyl acetate $(25 \mathrm{k} \Omega$ ) [88]. Despite the promising results, the authors did not clarify if the changes on electro resistance were specific nor if the phenomena were associated with the protein binding or with the structure of the film [88]. 
Biosensors for the control of wine and food quality Some authors have explored the potential of OBPs to create an electronic nose device (e-noses) [7,87]. The enoses are, by definition, electronic devices with the purpose of detecting odorant molecules [7]. These e-noses are similar to arrays where a sensing material is deposited in given support (e.g. gold, carbonnanotubes, siliconnitrate) and when a volatile organic compound (VOCs) interacts with this sensing material and generates a signal (e.g. electric, optical or gravimetric) that is transduced and gravimetrically processed [7]. These systems contain a transducer which can be based on the surface acoustic wave (SAW), surface plasmon resonance (SPR), or quartz crystal microbalances (QCMs), which generates a current-voltage that converts the biological events into a measurable signal $[7,86,87]$. SPR measures changes in the refractive index at the surface of the sensing element, while QCM are mass transducers and they measure very small mass changes, in the order of picograms [89]. Electronic-nose biosensors were tested to monitor the quality of wine, in terms of VOCs. The presence of specific functional groups defines the aroma and quality of wine [83]. The other applications of e-noses was on the detection of microbial toxins produced by human pathogens in foods. Using $\mathrm{pOBP}$ for the detection of R-(-)-1-octen-3-ol (octenol) and R-(-)-carvone in food samples, it is possible to correlate the presence of these compounds with the presence of fungi and molds [82]. The disadvantages of these systems are a low sensitivity (millimolar or ppm range) and the low selectivity, once that it can bind to several VOC molecules [7,30]. Further development of artificial chemosensory devices will be useful for food quality control, for the detection and progress evaluation of diseases, and for environmental security and agriculture monitoring [30,57].

\section{Biosensors for explosives and drugs detection}

The OBPs have also been reported as sensing elements for the detection of explosives. Manai et al. [90] reported the immobilization of pOBP onto diamond microcantilevers systems, which presented a good sensibility for the detection of 2,4-DNT, an analog of explosive TNT [90]. A preliminary investigation showed the potential of four OBPs for the detection of explosive components such as diphenylamine, dimethyl-phthalate, resorcinol, and dinitrotoluene [91]. Another approach was proposed by Cennamo et al. [84] that developed an optical biosensor based on the pOBP connected with surface plasmon resonance (SPR) transduction deposited in a plastic optical fiber, for the detection of butanal through a competitive assay. This device has a reduced cost and size and presents a detection range between 20 and $1000 \mu \mathrm{M}$ [84].

Dogs are commonly used to detect drugs, exogenous fruits, explosives, and other compounds. For these, they need to be trained and despite their excellent performances, some compounds are hardly detected. Rapid, sensitive, and selective biosensors can be a valid option, complementarity to the use of dogs [30]. Based on the ability of dogs to smell several odors with high sensitivity, D'Auria et al. [40] used a dog CfOBP (extracted from the animal nasal mucosa), to develop a biosensor based on refractive index measurements. Unfortunately, the experimental details of this study are scarce. The data showed little refractive index differences for CfOBP when exposed to pyrazine solution and pyrazine vapors, by comparison with a bovine serum albumin (BSA) response [40].

\section{Biosensors for medical diagnosis}

Differentiated biological ligands can be applied as potential biomarkers for the detection of cancer and other diseases. The immobilization of a human OBP on a nanopore array and its response to docosahexaenoic acid, lauric acid, and benzaldehyde, has been reported as a new biosensor with potential applications in the field of medical diagnosis. In this study, the authors used changes in electric impedance to detect and quantify these compounds [92]. More recently, it was described the potential of OBP-based sensors to screen volatile organic chemicals (VOCs) emitted by organisms, as components of noninvasive medical procedures, to monitor a patient's metabolic state or diagnose pathological conditions [30]. For example, a genetically encoded fluorescence resonance energy transfer (FRET)based nanosensor was described to monitor and quantify ethanol and other alcohols in living cells [93]. The nanosensor was designed using a human OBP ( $\mathrm{hOBP}_{\text {IIa }}$ ) flanked by two fluorescent proteins, enhanced cyan fluorescent protein (ECFP) and Venus at $\mathrm{C}$ and $\mathrm{N}$-terminus of OBP, respectively. The sensor revealed a dissociation constant (Kd) of $4.16 \mu \mathrm{M}$ for ethanol and due to its characteristics is a noninvasive sensing device with the potential to be used in bacteria, yeast, and mammalian cells [93].

\section{OBPs as capture and release devices of odorant molecules}

OBPs have shown to be ideal for the development of versatile sensing elements. Yet their applications can be further extended to other areas, like the capture of unpleasant odors and the programmed release of 
(a)

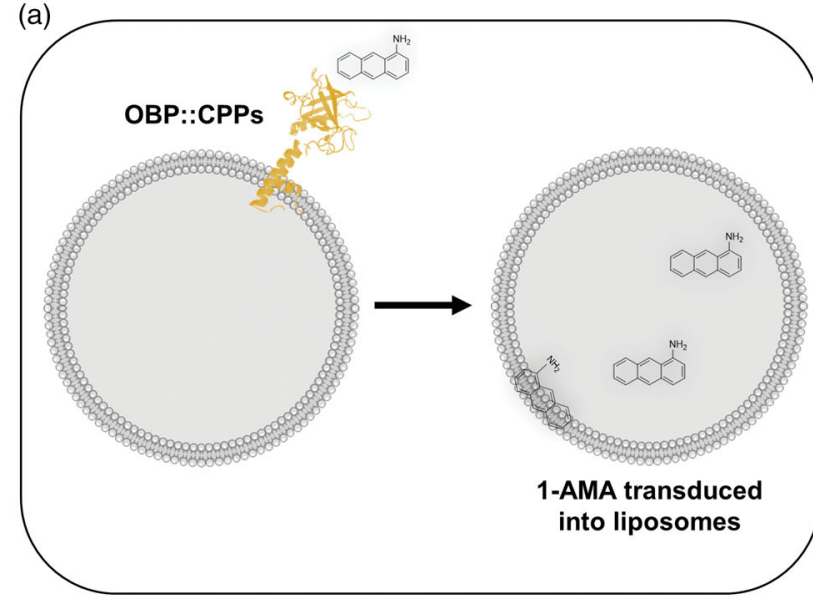

(b)

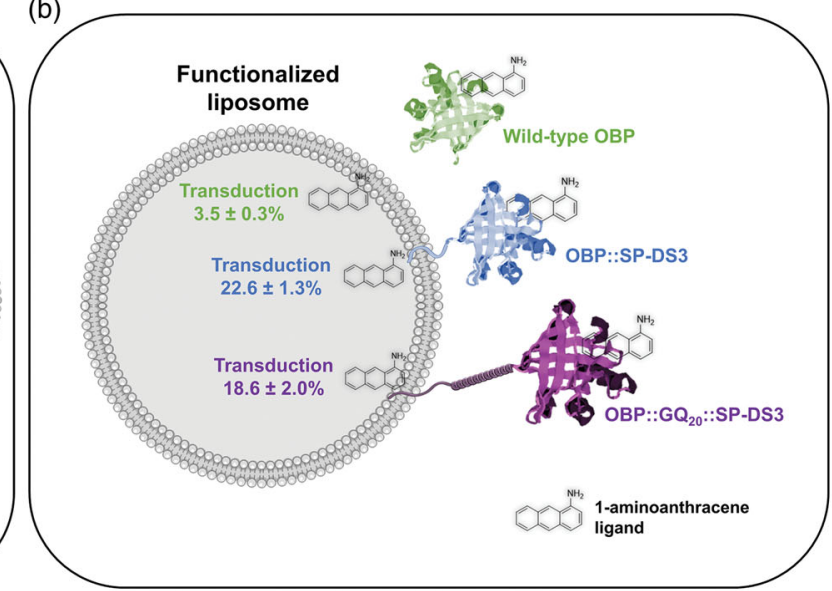

Figure 5. Applications of pig odorant-binding protein when conjugated with liposomes. (A) 1-aminoanthracene transduction by OBP fused with cell-penetrating peptides (OBP::CPPs) and by (B) OBP fused with anchor peptide SP-DS3, with or without $\mathrm{GQ}_{20}$ spacer (OBP::SP-DS3 and OBP::GQ $\mathrm{G}_{20}:: \mathrm{SP}-\mathrm{DS} 3$ ). These approaches can be used for the capture of molecules, for example, the capture of unpleasant or contaminant molecules. Figures based on/retrieved from Goncalves et al. [64,69].

fragrance molecules. In the study of Silva et al. [63], the authors explored the use of pig OBP (pOBP) functionalized onto cotton fabrics for the release of fragrances, and the reduction of unpleasant odors, such as cigarette smoke [63]. The authors evaluated the affinity of four fragrances ( $\beta$-citronellol, citronellyl valerate, ethyl valerate, and benzyl benzoate) to pOBP by competitive assay, with the $\beta$-citronellol presenting the highest affinity [63]. After functionalization of cationized cotton fabrics (chemical reaction of cationic reactive agents with cellulose to impart positive charges to the cotton surface) with the pOBP/citronellol complex (1:2 molar proportion), the release of $\beta$-citronellol was evaluated at $37^{\circ} \mathrm{C}$ by headspace gas chromatography-mass spectrometry (HS-GC-MS). After $5 \mathrm{~min}$ at $37^{\circ} \mathrm{C}$, a release of around $42 \%$ of the initial concentration of fragrance was detected [63]. A human panel evaluated the cigarette smell in samples of cotton functionalized with pOBP and verified a higher reduction of the smoke smell when compared with non-functionalized cotton samples [63]. This research opened the potential of OBPs as devices for the capture of odors and for the release of fragrances and other molecules (antimicrobial agents and insect repellents), with high interest for the textile and cosmetic industries.

Pig OBP was fused to three cell-penetrating peptides (CPPs), named Tat, Pep-1 and pVEC. When added to liposomes, these new fusion proteins revealed different transduction efficiencies of a model ligand (1-aminoanthracene, 1-AMA) into liposomes (Figure 5) [64]. The highest 1-AMA transduction efficiency was obtained for the OBP::Tat fusion protein ( $42 \%)$. The transduction of 1-AMA has shown to be dependent on the amino acid sequence of CPP, its charge, and its hydrophobic character [64]. Two further fusion proteins, pOBP fused with the anchor peptide SP-DS3 [94] with or without a spacer $\left(\mathrm{GQ}_{20}\right)$, were obtained by Gonçalves et al. [65]. The effect of the protein proximity (absence or presence of $\mathrm{GQ}_{20}$ spacer) on the 1-AMA transduction into liposomes was evaluated by producing liposomes with the fusion proteins anchored in the lipidic membrane. The study demonstrated that the transduction of the 1AMA ligand into liposomes is driven by the proximity of the protein to liposomal membranes. The presence of the spacer promoted higher binding of 1-AMA to the protein ( $\sim 45 \%$ for $\mathrm{OBP}:: \mathrm{GQ}_{20} 0: \mathrm{SP}-\mathrm{DS} 3$ and $\sim 29 \%$ for OBP::SP-DS3) but decreased the transduction of 1-AMA into the liposome ( $19 \%$ for OBP:::GQ $\mathrm{GQ}_{20}:: \mathrm{SP}-\mathrm{DS} 3$ and $\sim 23 \%$ for OBP::SP-DS3) [65]. These systems further explored the potential of engineered OBPs for the retention of odors in proteins and/or into liposomes. This work highlights the use of OBP-functionalized liposomes as devices for the encapsulation of molecules, used for textile and cosmetic industries.

\section{OBPs as thermo-responsive proteins}

Taking advantage of their high thermal stability and the ability to accommodate and release differentiated ligands, OBPs have been explored for the development of thermo-responsive systems. The structure of two recombinant proteins based on the pOBP sequence truncated $\mathrm{OBP}$ and the $\mathrm{OBP}:: \mathrm{GQ}_{20}:: \mathrm{SP}-\mathrm{DS} 3$, were predicted by molecular modeling simulations. The truncated OBP (tOBP-F44A/F66A) was obtained from the deletion of the first 16 residues of the $\mathrm{N}$-terminal and the replacement of two phenylalanine residues at the binding pocket by alanine residues (F44A and F66A). 


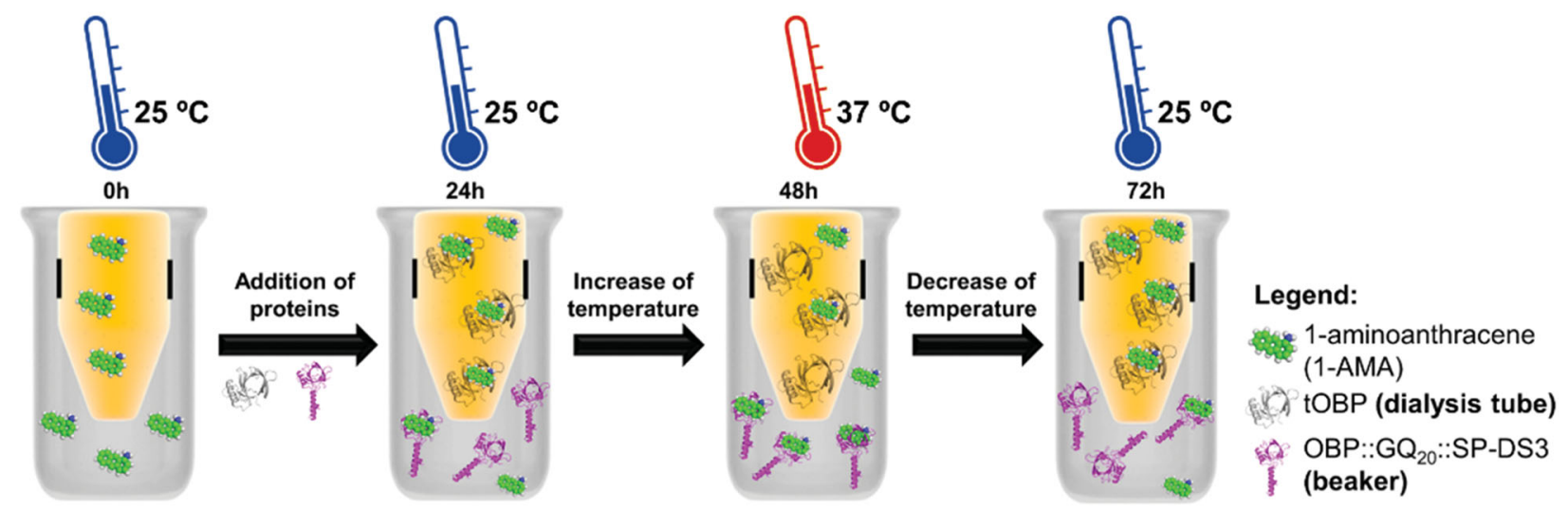

Figure 6. Opposite binding behavior of truncated OBP (tOBP-F44A/F66A) and OBP::GQ20::SP-DS3. The engineered pig OBPs were separated in a beaker by a dialysis membrane with a cutoff permeable to 1-AMA (3.5 kDa). tOBP was placed inside the dialysis tube and the OBP::GQ20::SP-DS3 was placed outside. After equilibrating the 1-AMA concentration in both compartments, the proteins were added and the temperature was alternated between 25 and $37^{\circ} \mathrm{C}$. The binding in both compartments was measured for each temperature. The figure is based on Goncalves et al. [64].

The OBP::GQ $\mathrm{GQ}_{20}:: \mathrm{SP}-\mathrm{DS} 3$ resulted from the fusion of pOBP with a spacer of 20 repetitions of glycine-glutamine residues, to promote molecular mobility, and the anchor peptide SP-DS3 [69]. Using 1-AMA as a model ligand, it was found to be a differentiated binding preference toward the OBPs depending on the temperature $\left(25\right.$ and $37^{\circ} \mathrm{C}$ ). Experimental data showed that 1-AMA binds preferentially to tOBP-F44A/F66A at $25^{\circ} \mathrm{C}$, while it is binding to OBP:: $\mathrm{GQ}_{20}$ ::SP-DS3 was favored at $37^{\circ} \mathrm{C}$ (Figure 6). These experimental pieces of evidence were confirmed by molecular modeling studies. The variation of the OBPs' binding pocket size and the position of 1AMA during the binding process was elucidated for both temperatures. The size of the binding pocket was determinant for the binding preference of 1-AMA, with higher bindings being obtained when the binding pocket was larger. The AutoDock tool allowed the identification of the OBPs' amino acid residues involved in the binding of 1-AMA. Different residues were identified according to the binding temperature, of which some were already reported for the wild-type pOBP, while others were described for the first time [69]. The elucidation of the mechanism associated with the binding preference of 1-AMA to different OBPs depending on the temperature established the use of OBPs in the design of temperature-dependent biosensors.

\section{Release of fragrances from OBP triggered by perspiration - the effect of saline solutions}

OBPs are carrier proteins that might be responsible for the transport of insoluble odorant molecules across the nasal mucus into the olfactory receptors. The mechanism by which the recognition of the odorant molecules is conducted is still unknown. However, it is suggested that the mechanism of odorants recognition is based on the interaction between the OBP/odorant complex and the olfactory receptor [38,95]. After recognition, the odorant molecule dissociates from the OBP, and the protein is again available to bind a new odorant molecule. The dissociation of the fragrance can be dictated by several parameters like molecule competition mechanisms for the OBPs binding pocket, the variation of temperature and $\mathrm{pH}$, presence of organic solvents, or variation of the ionic strength $[56,96]$. We have found that the release of fragrances from the OBPs is triggered in the presence of saline solutions (artificial sweat $\mathrm{pH}$ 4.3-8.5). Using pig OBP we verified that the release of 1-AMA and $\beta$-citronellol molecules, at $37^{\circ} \mathrm{C}$, was promoted by an artificial sweat solution. Comparing the association constants $(\mathrm{Ka})$ in the presence of salty and unsalted solutions, a decrease in the Ka was noticed for both molecules. For 1-AMA a $\mathrm{Ka}=4.00 \pm 0.03 \mu \mathrm{M}$ and a $\mathrm{Ka}=0.20 \pm 0.02 \mu \mathrm{M}$ was obtained, for unsalted and salty solutions, respectively. For the $\beta$-citronellol the same tendency was observed, with a $\mathrm{Ka}=$ $2.50 \pm 0.03 \mu \mathrm{M}$ for unsalted conditions and a $\mathrm{Ka}=$ $0.20 \pm 0.02 \mu \mathrm{M}$ for salty conditions. The dissociation mechanism promoted changes in the salinity of the OBPs surrounding milieu, lead us to formulate an IP regarding the effect of saline solutions (perspiration) on the release of fragrances for cosmetic applications (INPI n 116561$)$.

Recently, based on this dissociation mechanism, we developed a smart responsive textile functionalized with an engineered pig OBP - OBP:: $\mathrm{GQ}_{20}:: \mathrm{CBM}$. This protein was obtained by the fusion of $\mathrm{POBP}$ with a glycine-glutamine spacer $\left(\mathrm{GQ}_{20}\right)$ and a carbohydrate-binding module (CBM). Through fusion with the $C_{B M}$ (PDB ID: 1ULP) from endoglucanase $C$ from 


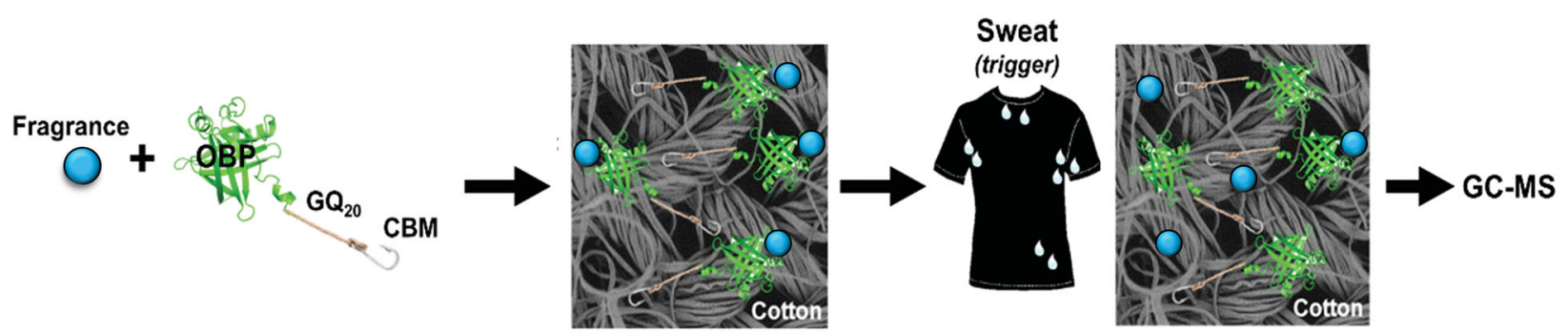

Figure 7. Fragrance release from $\mathrm{OBP}:: \mathrm{GQ}_{20}:: \mathrm{CBM}$ functionalized on cotton, against perspiration. The release of fragrance was measured by GC-MS. Figure was retrieved from Goncalves et al. [66].

Cellulomonas fimi, the OBP acquired a specific affinity to cotton. The textile, functionalized with the $\mathrm{OBP}:: \mathrm{GQ}_{20}:: \mathrm{CBM}$, exhibited the ability to release $\beta$-citronellol in response to perspiration. When the textile was exposed to a sweat saline solution, the release of the fragrance, measured after $1.5 \mathrm{~h}$, was around $32 \%$ (Figure 7). The sweat saline solution modified the intermolecular interactions involved in the ligand-OBP complex triggering the release of the fragrance, indicating that this mechanism can be further explored in the development of responsive devices for daily activity and physical exercise [66]. Furthermore, these results allowed us to predict the successful exploitation of this protein in cosmetic applications.

\section{Major remarks and future prospects}

Odor perception is a vital process for animals in general. It helps the animals in the identification of members from the same or from different species, the identification of food and potential poisons [3-5]. The olfactory events involve the interactions of odorants with the olfactory receptors assisted by the OBPs. Although the mechanisms of interaction between the OBP and ligands, and of the OBP/ligand complex with the ORs are still not well understood, it has been demonstrated that OBPs contribute to olfactory perception at several levels: transport, prevention of oxidative stress, and prevention of ORs saturation. Mammalian OBPs act as passive carriers of hydrophobic odorant molecules from the environment to the olfactory receptors $[1,9,12,77]$. The $\beta$-barrel structure of OBPs and their ability to bind different types of molecules, suggests their potential role in vertebrates' olfactory system.

The total knowledge of the olfactory system and the mechanisms involved in smell perception are still scarce, mostly due to the complexity of the mechanisms involved, from the molecular aspects of the odorant bindings to the final signal transduction to the limbic system. It is crucial to understand the molecular mechanisms involved in the association and dissociation between the ligand and the OBP, and between the OBP/ligand complex with the OR.

Ligand-binding experiments allow characterizing OBPs, their role in chemical communication, and their capacity to associate/dissociate to different molecules. With advances in informatics tools, molecular dynamic simulations and molecular docking experiments have provided detailed information about the nature of OBP/ ligand interactions and the position of ligands in the proteins. Genomics and proteomics have allowed us to obtain more information about the identification and function of OBPs. These new tools can also help the identification of new OBPs and their function. For example, some binding proteins were identified in databases as OBPs but the main ligands are pheromones; thus, this proteins' classification would be renamed as pheromone-binding protein. The outstanding stability of OBPs to thermal denaturation, $\mathrm{pH}$, and proteolytic degradation make these proteins excellent candidates for the development of sensing devices for: pollution control, agriculture, healthcare, security, cosmetic, and food industry applications $[29,30,83]$. Furthermore, OBP is involved in smell perception and some studies suggest that it could be used for odor control and the controlled release of fragrances from a textile $[63,66]$.

The utilization of OBPs in several reported fields are dependent on social and economic impacts, and on the advantages (solubility, stability, detection of different molecules) and disadvantages (low selectivity and sensitivity) associated.

\section{Disclosure statement}

No potential conflict of interest was reported by the author(s).

\section{Funding}

This study was financially supported by the Portuguese Foundation for Science and Technology (Fundação para a Ciência e a Tecnologia) under the scope of the strategic funding of UID/BIO/04469/2019 unit and BioTecNorte 
operation [NORTE-01-0145-FEDER-000004] funded by European Regional Development Fund under the scope of Norte2020 - Programa Operacional Regional do Norte. F.G. thanks Fundacão para a Ciência e a Tecnologia for their funding [SFRH/BD/114684/2016].

\section{ORCID}

Filipa Gonçalves (iD http://orcid.org/0000-0002-1928-7975

Artur Ribeiro (D) http://orcid.org/0000-0003-3298-5564

Carla Silva (D http://orcid.org/0000-0001-6252-8693

Artur Cavaco-Paulo iD http://orcid.org/0000-0001-7204-2064

\section{References}

[1] Breer H. Olfactory receptors: molecular basis for recognition and discrimination of odors. Anal Bioanal Chem. 2003;377(3):427-433.

[2] Menini A, Lagostena L, Boccaccio A. Olfaction: from odorant molecules to the olfactory cortex. News Physiol Sci. 2003;19:101-104.

[3] Alfinito E, Millithaler JF, Pennetta C, et al. A single protein based nanobiosensor for odorant recognition. Microelectron J. 2010;41(11):718-722.

[4] Archunan G. Odorant binding proteins: a key player in the sense of smell. Bioinformation. 2018;14(1):36-37.

[5] Muthukumar S, Rajesh D, Selvam RM, et al. Buffalo nasal odorant-binding protein (bunOBP) and its structural evaluation with putative pheromones. Sci Rep. 2018;8(1):9323.

[6] Pelosi P. Odorant-binding proteins: structural aspects. Ann NY Acad Sci. 1998;855(1):281-293.

[7] Barbosa AJM, Oliveira AR, Roque ACA. Protein- and Peptide-Based Biosensors in Artificial Olfaction. Trends Biotechnol. 2018;36(12):1244-1258.

[8] de March CA, Kim SK, Antonczak S, et al. G proteincoupled odorant receptors: from sequence to structure. Protein Sci. 2015;24(9):1543-1548.

[9] Firestein S. How the olfactory system makes sense of scents. Nature. 2001;413(6852):211-218.

[10] Lee JY, Ko HJ, Lee SH, et al. Cell-based measurement of odorant molecules using surface plasmon resonance. Enzyme Microb Technol. 2006;39(3):375-380.

[11] Zhou G, Lane G, Cooper SL, et al. Characterizing functional pathways of the human olfactory system. eLife. 2019;8:e47177.

[12] Sankaran S, Khot LR, Panigrahi S. Biology and applications of olfactory sensing system: a review. Sens Actuators, B. 2012;171-172:1-17.

[13] Flower DR. The lipocalin protein family: structure and function. Biochem J. 1996;318(1):1-14.

[14] Flower DR. Beyond the superfamily: the lipocalin receptors. Biochim Biophys Acta. 2000;1482(1-2): 327-336.

[15] Borysik AJ, Briand L, Taylor AJ, et al. Rapid odorant release in mammalian odour binding proteins facilitates their temporal coupling to odorant signals. J Mol Biol. 2010;404(3):372-380.

[16] Gomez-Velasco H, Rojo-Dominguez A, GarciaHernandez E. Enthalpically-driven ligand recognition and cavity solvation of bovine odorant binding protein. Biophys Chem. 2020;257:106315.

[17] Pelosi P. The role of perireceptor events in vertebrate olfaction. Cell Mol Life Sci. 2001;58(4):503-509.

[18] Krishna NSR, Getchell ML, Margolis FL, et al. Differential expression of vomeromodulin and odorant-binding protein, putative pheromone and odorant transporters, in the developing rat nasal chemosensory mucosae. J Neurosci Res. 1995;40(1):54-71.

[19] Bignetti E, Cavaggioni A, Pelosi P, et al. Purification and characterisation of an odorant-binding protein from cow nasal tissue. Eur J Biochem. 1985;149(2): 227-231.

[20] Dal Monte M, Andreini I, Revoltella R, et al. Purification and characterization of two odorant-binding proteins from nasal tisue of rabbit and pig. Comp Biochem Physiol. 1991;99(2):445-451.

[21] Garibotti M, Navarrini A, Pisanelli AM, et al. Three odorant-binding proteins from rabbit nasal mucosa. Chem Senses. 1997;22(4):383-390.

[22] Lazar J, Greenwood DR, Rasmussen LEL, et al. Molecular and functional characterization of an odorant binding protein of the asian elephant, Elephas maximus: implications for the role of lipocalins in mammalian olfaction. Biochemistry. 2002;41(39): 11786-11794.

[23] Pes D, Dal Monte M, Ganni M, et al. Isolation of two odorant-binding proteins from mouse nasal tissue. Comp Biochem Physiol B. 1992;103(4):1011-1017.

[24] Lobel D, Strotmann J, Jacob $M$, et al. Identification of a third rat odorant-binding protein (OBP3). Chem Senses. 2001;26(6):673-680.

[25] Fan J, Francis F, Liu Y, et al. An overview of odorantbinding protein functions in insect peripheral olfactory reception. Genet Mol Res. 2011;10(4):3056-3069.

[26] Lee K-H, Wells RG, Reed RR. Isolation of an olfactory CDNA: similarity to retinol-binding protein suggests a role in olfaction. Science. 1987;235(4792):1053-1056.

[27] Briand L, Eloit C, Nespoulous C, et al. Evidence of an odorant-binding protein in the human olfactory mucus: location, structural characterization, and odorant-binding properties. Biochemistry. 2002;41(23): 7241-7252.

[28] Tegoni $M$, Pelosi $P$, Vincent $F$, et al. Mammalian odorant binding proteins. Biochim Biophys Acta. 2000; 1482(1-2):229-240.

[29] Pelosi P, Mastrogiacomo R, lovinella I, et al. Structure and biotechnological applications of odorant-binding proteins. Appl Microbiol Biotechnol. 2014;98(1):61-70.

[30] Cave JW, Wickiser JK, Mitropoulos AN. Progress in the development of olfactory-based bioelectronic chemosensors. Biosens Bioelectron. 2019;123:211-222.

[31] Bignetti $E$, Cattaneo $P$, Cavaggioni $A$, et al. The pyrazine-binding protein and olfaction. Comp Biochem Physiol B. 1988;90(1):1-5.

[32] Pevsner J, Hou V, Snowman AM, et al. Odorant-binding protein: characterization of ligand binding. J Biol Chem. 1990;265(11):6118-6125.

[33] Grolli S, Merli E, Conti V, et al. Odorant binding protein has the biochemical properties of a scavenger for 4-hydroxy-2-nonenal in mammalian nasal mucosa. FEBS J. 2006;273(22):5131-5142. 
[34] Ikematsu M, Takaoka D, Yasuda M. Odorant binding initially occurring at the central pocket in bovine odorant-binding protein. Biochem Biophys Res Commun. 2005;333(4):1227-1233.

[35] Kiefer $H$, Krieger J, Olszewski JD, et al. Expression of an olfactory receptor in Escherichia coli: purification, reconstitution, and ligand binding. Biochemistry. 1996; 35(50):16077-16084.

[36] Malnic B, Hirono J, Sato T, et al. Combinatorial receptor codes for odors. Cell. 1999;96(5):713-723.

[37] Vidic J, Grosclaude J, Monnerie R, et al. On a chip demonstration of a functional role for odorant binding protein in the preservation of olfactory receptor activity at high odorant concentration. Lab Chip. 2008;8(5):678-688.

[38] Matarazzo V, Zsurger N, Guillemot J-C, et al. Porcine odorant-binding protein selectively binds to a human olfactory receptor. Chem Senses. 2002;27(8):691-701.

[39] White SA, Briand L, Scott DJ, et al. Structure of rat odorant-binding protein OBP1 at $1.6 \mathrm{~A}$ resolution. Acta Crystallogr D Biol Crystallogr. 2009;65(5):403-410.

[40] D'Auria S, Staiano M, Varriale A, et al. The odorantbinding protein from Canis familiaris: purification, characterization and new perspectives in biohazard assessment. Protein Pept Lett. 2006;13(4):349-352.

[41] Lobel D, Marchese S, Krieger J, et al. Subtypes of odorant-binding proteins-heterologous expression and ligand binding. Eur J Biochem. 1998;254(2):318-324.

[42] Heydel JM, Coelho A, Thiebaud N, et al. Odorant-binding proteins and xenobiotic metabolizing enzymes: implications in olfactory perireceptor events. Anat Rec. 2013;296(9):1333-1345.

[43] Lacazette E, Gachon AM, Pitiot G. A novel human odorant-binding protein gene family resulting from genomic duplicons at 9q34: differential expression in the oral and genital spheres. Hum Mol Genet. 2000; 9(2):289-301.

[44] Cunningham F, Achuthan P, Akanni W, et al. Ensembl 2019. Nucleic Acids Res. 2019;47(D1):D745-D751.

[45] Tegoni M, Ramoni R, Bignetti E, et al. Domain swapping creates a third putative combining site in bovine odorant binding protein dimer. Nat Struct Biol. 1996; 3(10):863-867.

[46] Bianchet MA, Bains G, Pelosi P, et al. The three-dimensional structure of bovine odorant binding protein and its mechanism of odor recognition. Nat Struct Biol. 1996;3(11):934-939.

[47] Spinelli S, Ramoni R, Grolli S, et al. The structure of the monomeric porcine odorant binding protein sheds light on the domain swapping mechanism. Biochemistry. 1998;37(22):7913-7918.

[48] Vincent F, Spinelli S, Ramoni R, et al. Complexes of porcine odorant binding protein with odorant molecules belonging to different chemical classes. J Mol Biol. 2000;300(1):127-139.

[49] Schiefner A, Freier R, Eichinger A, et al. Crystal structure of the human odorant binding protein, OBPIla. Proteins. 2015;83(6):1180-1184.

[50] Pelosi P. Odorant-binding proteins: structural aspects. Annals NY Acad Sci. 1998;855(1):281-293.
[51] Perduca M, Mancia F, Del Giorgio R, et al. Crystal structure of a truncated formof porcine odorant-binding protein. Proteins. 2001;42(2):201-209.

[52] Paolini S, Tanfani F, Fini C, et al. Porcine odorant-binding protein: structural stability and ligand afinities measured by Fourier-transform infrared spectroscopy and fluorescence spectroscopy. Biochimica et Biophysica Acta. 1999;1431:179-188.

[53] Staiano M, D'Auria S, Varriale A, et al. Stability and dynamics of the porcine odorant-binding protein. Biochemistry. 2007;46(39):11120-11127.

[54] Stepanenko OV, Roginskii DO, Stepanenko OV, et al. Structure and stability of recombinant bovine odorant-binding protein: I. Design and analysis of monomeric mutants. PeerJ. 2016;4:e1933.

[55] Ramoni R, Vincent F, Ashcroft A, et al. Control of domain swapping in bovine odorant-binding protein. Biochem J. 2002;365(3):739-748.

[56] Mazzini A, Polverini E, Parisi M, et al. Dissociation and unfolding of bovine odorant binding protein at acidic pH. J Struct Biol. 2007;159(1):82-91.

[57] Capo A, Pennacchio A, Varriale A, et al. The porcine odorant-binding protein as molecular probe for benzene detection. PLoS One. 2018;13(9):e0202630.

[58] Stepanenko OV, Stepanenko OV, Staiano M, et al. The quaternary structure of the recombinant bovine odorant-binding protein is modulated by chemical denaturants. PLoS One. 2014;9(1):e85169.

[59] Burova TV, Choiset Y, Jankowski CK, et al. Conformational stability and binding properties of porcine odorant binding protein. Biochemistry. 1999; 38(45):15043-15051.

[60] Pelosi P, Baldaccini NE, Pisanelli AM. Identification of a specific olfactory receptor for 2-isobutyl-3-methoxypyrazine. Biochem J. 1982;201(1):245-248.

[61] Pelosi P, Zhu J, Knoll W. From radioactive ligands to biosensors: binding methods with olfactory proteins. Appl Microbiol Biotechnol. 2018;102(19):8213-8227.

[62] Albani JR, Bretesche L, Vogelaer J, et al. Energy transfer studies between Trp residues of three lipocalin proteins family, $\alpha 1$-acid glycoprotein, (orosomucoid), $\beta$-lactoglobulin and porcine odorant binding protein and the fluorescent probe, 1-aminoanthracene (1AMA). J Fluoresc. 2015;25(1):167-172.

[63] Silva C, Matama T, Azoia NG, et al. Odorant binding proteins: a biotechnological tool for odour control. Appl Microbiol Biotechnol. 2014;98(8):3629-3638.

[64] Goncalves F, Castro TG, Nogueira E, et al. OBP fused with cell-penetrating peptides promotes liposomal transduction. Colloids Surf B Biointerfaces. 2018;161: 645-653.

[65] Gonçalves F, Silva C, Ribeiro A, et al. 1-aminoanthracene transduction into liposomes driven by odorantbinding protein proximity. ACS Appl Mater Interfaces. 2018;10(32):27531-27539.

[66] Goncalves F, Ribeiro A, Silva C, et al. Release of fragrances from cotton functionalized with carbohydrate-binding module proteins. ACS Appl Mater Interfaces. 2019;11(31):28499-28506.

[67] Seo $\mathrm{MH}$, Park J, Kim E, et al. Protein conformational dynamics dictate the binding affinity for a ligand. Nat Commun. 2014;5:3724. 
[68] Mastrogiacomo R, lovinella I, Napolitano E. New fluorescent probes for ligand-binding assays of odorantbinding proteins. Biochem Biophys Res Commun. 2014;446(1):137-142.

[69] Goncalves F, Castro TG, Azoia NG, et al. Two Engineered OBPs with opposite temperature-dependent affinities towards 1-aminoanthracene. Sci Rep. 2018;8(1):14844.

[70] Wei Y, Brandazza A, Pelosi P. Binding of polycyclic aromatic hydrocarbons to mutants of odorant-binding protein: a first step towards biosensors for environmental monitoring. Biochim Biophys Acta. 2008; 1784(4):666-671.

[71] Marabotti A, Lefevre T, Staiano $M$, et al. Mutant bovine odorant-binding protein: temperature affects the protein stability and dynamics as revealed by infrared spectroscopy and molecular dynamics simulations. Proteins. 2008;72(2):769-778.

[72] Stepanenko OV, Marabotti A, Kuznetsova IM, et al. Hydrophobic interactions and ionic networks play an important role in thermal stability and denaturation mechanism of the porcine odorant-binding protein. Proteins. 2008;71(1):35-44.

[73] Stepanenko O, Fonin A, Stepanenko O, et al. Ligandbinding proteins: structure, stability and practical application. In: Faraggi DE, editor. Protein structure. London (UK): InTech; 2012. p. 265-290.

[74] Meillour PN, Lagant P, Cornard JP, et al. Phenylalanine 35 and tyrosine 82 are involved in the uptake and release of ligand by porcine odorant-binding protein. Biochim Biophys Acta. 2009;1794(8):1142-1150.

[75] Golebiowski J, Antonczak S, Fiorucci S, et al. Mechanistic events underlying odorant binding protein chemoreception. Proteins. 2007;67(2):448-458.

[76] Kmiecik D, Albani JR. Effect of 1-aminoanthracene (1AMA) binding on the structure of three lipocalin proteins, the dimeric $\beta$ lactoglobulin, the dimeric odorant binding protein and the monomeric $\alpha 1$-acid glycoprotein. Fluorescence spectra and lifetimes studies. J Fluoresc. 2010;20(5):973-983.

[77] Hajjar E, Perahia D, Debat $\mathrm{H}$, et al. Odorant binding and conformational dynamics in the odorant-binding protein. J Biol Chem. 2006;281(40):29929-29937.

[78] Tcatchoff L, Nespoulous C, Pernollet JC, et al. A single lysyl residue defines the binding specificity of a human odorant-binding protein for aldehydes. FEBS Lett. 2006;580(8):2102-2108.

[79] Zhu J, Arena S, Spinelli S, et al. Reverse chemical ecology: olfactory proteins from the giant panda and their interactions with putative pheromones and bamboo volatiles. Proc Natl Acad Sci USA. 2017;114(46): E9802-E9810.

[80] Venthur H, Mutis A, Zhou J-J, et al. Ligand binding and homology modelling of insect odorant-binding proteins. Physiol Entomol. 2014;39(3):183-198.

[81] Golebiowski J, Antonczak S, Cabrol-Bass D. Molecular dynamics studies of odorant binding protein free of ligand and complexed to pyrazine and octenol. J Mol Struct Theochem. 2006;763(1-3):165-174.

[82] Di Pietrantonio F, Cannata D, Benetti $M$, et al. Detection of odorant molecules via surface acoustic wave biosensor array based on odorant-binding proteins. Biosens Bioelectron. 2013;41:328-334.

[83] Wilson AD. Diverse applications of electronic-nose technologies in agriculture and forestry. Sensors. 2013;13(2):2295-2348.

[84] Cennamo N, Di Giovanni S, Varriale A, et al. Easy to use plastic optical fiber-based biosensor for detection of butanal. PLoS One. 2015;10(3):e0116770.

[85] Bianchi F, Basini G, Grolli S, et al. An innovative bovine odorant binding protein-based filtering cartridge for the removal of triazine herbicides from water. Anal Bioanal Chem. 2013;405(2-3):1067-1075.

[86] Di Pietrantonio F, Benetti M, Cannata D, et al. A surface acoustic wave bio-electronic nose for detection of volatile odorant molecules. Biosens Bioelectron. 2015;67:516-523.

[87] Mulla MY, Tuccori E, Magliulo M, et al. Capacitancemodulated transistor detects odorant binding protein chiral interactions. Nat Commun. 2015;6:6010.

[88] Hou Y, Jaffrezic-Renault N, Martelet C, et al. Study of Langmuir and Langmuir-Blodgett films of odorantbinding protein/amphiphile for odorant biosensors. Langmuir. 2005;21(9):4058-4065.

[89] Persaud KC, Tuccori E. Biosensors based on odorant binding proteins. In: Park T, editor. Bioelectronic nose. Dordrecht (The Netherlands): Springer; 2014. p. 171-190.

[90] Manai R, Scorsone E, Rousseau L, et al. Grafting odorant binding proteins on diamond bio-MEMS. Biosens Bioelectron. 2014;60:311-317.

[91] Ramoni R, Bellucci S, Grycznyski I, et al. The protein scaffold of the lipocalin odorant-binding protein is suitable for the design of new biosensors for the detection of explosive components. J Phys: Condens Matter. 2007;19(39):395012.

[92] Lu Y, Zhang D, Zhang Q, et al. Impedance spectroscopy analysis of human odorant binding proteins immobilized on nanopore arrays for biochemical detection. Biosens Bioelectron. 2016;79:251-257.

[93] Soleja N, Manzoor O, Nandal P, et al. FRET-based nanosensors for monitoring and quantification of alcohols in living cells. Org Biomol Chem. 2019;17(9): 2413-2422.

[94] Nogueira E, Mangialavori IC, Loureiro A, et al. Peptide anchor for folate-targeted liposomal delivery. Biomacromolecules. 2015;16(9):2904-2910.

[95] Pelosi P. Odorant-binding proteins. Crit Rev Biochem Mol Biol. 1994;29(3):199-228.

[96] Parisi M, Mazzini A, Sorbi RT, et al. Unfolding and refolding of porcine odorant binding protein in guanidinium hydrochloride: equilibrium studies at neutral pH. Biochim Biophys Acta. 2003;1652(2):115-125. 